\title{
Study of absorption and re-emission processes in a ternary liquid scintillation system*
}

\author{
XIAO Hua-Lin(肖华林 $)^{1 ; 1)}$ LI Xiao-Bo(李小波 $)^{2} \quad$ ZHENG Dong(郑东 $)^{3}$ \\ CAO Jun(曹俊) ${ }^{2}$ WEN Liang-Jian(温良剑) ${ }^{2} \quad$ WANG Nai-Yan(王乃彦) ${ }^{1}$ \\ ${ }^{1}$ College of Nuclear Science and Technology, Beijing Normal University, Beijing 100875, China \\ ${ }^{2}$ Institute of High Energy Physics, Chinese Academy of Sciences, Beijing 100049, China \\ 3 Analytical and Testing Center, Beijing Normal University, Beijing 100875, China
}

\begin{abstract}
Liquid scintillators are widely used as the neutrino target in neutrino experiments. The absorption and emission of different components of a ternary liquid scintillator (Linear Alkyl Benzene (LAB) as the solvent, 2,5-diphenyloxazole (PPO) as the fluor and p-bis-(o-methylstyryl)-benzene (bis-MSB) as wavelength shifter) are studied. It is shown that the absorption of this liquid scintillator is dominant by LAB and PPO at wavelengths less than $349 \mathrm{~nm}$, and the absorption by bis-MSB becomes prevalent at the wavelength larger than $349 \mathrm{~nm}$. The fluorescence quantum yields, which are the key parameters to model the absorption and re-emission processes in large liquid scintillation detectors, are measured.
\end{abstract}

Key words liquid scintillator, absorption and re-emission, fluorescence quantum yield

PACS 29.40.Mc

\section{Introduction}

Liquid scintillators have been used extensively in neutrino experiments. Recently, a ternary liquid scintillator has been proposed for the Daya Bay Reactor Neutrino Experiment [1-3]. LAB is chosen as the solvent, and $3 \mathrm{~g} / \mathrm{L} \mathrm{PPO}$ and $15 \mathrm{mg} / \mathrm{L}$ bis-MSB are chosen as the primary solute and the secondary solute, respectively. The liquid scintillator doped with $0.1 \%$ Gadolinium will be used as the neutrino target and the undoped one will be used as the Gamma-Catcher. Some properties of this ternary liquid scintillator have been studied in Refs. [2, 4-6]. In this paper, we will study the absorption and emission properties.

When a liquid scintillation solution is exposed to ionizing radiation, the primary radiation energy is primarily absorbed mostly by the solvent which makes up the bulk of the solution, then partially transferred to the solute via two processes. One is a non-radiative process, which involves the dipoledipole interaction and the short-distance collision between the donor and acceptor molecules when they come sufficiently close to each other. The other is a radiative process, which involves the absorption and re-emission of the photons $[7,8]$. In a ternary liquid scintillator, the primary solute is always utilized as a fluor in order to obtain a higher scintillation efficiency and the secondary solute is always utilized as the wavelength shifter in order to minimize the selfabsorption of the scintillation and provide a better match between the photomultiplier spectral response and the fluorescence emission spectrum. Generally, the fluorescence efficiency of the solvent itself is small [7]. The radiative energy transfer process between the solvent and the fluor is insignificant and most of the excitation energy is migrated to the fluor by a nonradiative process. Hence, most of the scintillation is primarily generated by the fluor.

Both of scintillation generated by the fluor and the Cerenkov photons produced by charge particles could be absorbed and the photons can be re-emitted by the liquid scintillator, which makes the scintillation spectrum red-shift [9] and modifies the time profile of the scintillation [10]. It is important to model the optical process to study the behaviors of the scintillation detector. In this work, the absorptions of different

Received 25 January 2010

* Supported by Nation Natural Science Foundation of China (211202037)

1) E-mail:xiaohl@mail.bnu.edu.cn

(C)2010 Chinese Physical Society and the Institute of High Energy Physics of the Chinese Academy of Sciences and the Institute of Modern Physics of the Chinese Academy of Sciences and IOP Publishing Ltd 
components of this ternary liquid scintillator and their emission spectra are studied. The fluorescence quantum yields, which are defined as the re-emission probability after an absorption by the PPO and the bis-MSB, are measured.

\section{Absorption and emission}

The absorption cross section $\sigma$ can be expressed as $1 / \Lambda$, where $\Lambda$ is the optical attenuation length, which is defined as the distance at which the incident light intensity $I_{0}$ reduces to its $1 / e$. The attenuation length $\Lambda$ can be calculated by

$$
I(d) / I_{0}=\mathrm{e}^{-d / \Lambda},
$$

where $I_{0}$ is the original light intensity and $I(d)$ is the light intensity after a path $d$. The attenuation length $\Lambda$ can be obtained by measuring the transmission, i.e. $I(d) / I_{0}$, by an ultraviolet spectroscopy method.

In order to study the attenuations of different components in the ternary liquid scintillator, we prepared four samples. Their recipes are listed in Table 1. The attenuation length measurements were performed by a double beam UV-Vis spectrophotometer.

Table 1. Samples in the attenuation length measurement.

\begin{tabular}{cccc}
\hline sample & solvent & PPO & bis-MSB \\
\hline A & LAB & 0 & 0 \\
B & LAB & $3 \mathrm{~g} / \mathrm{L}$ & 0 \\
C & LAB & 0 & $15 \mathrm{mg} / \mathrm{L}$ \\
D & LAB & $3 \mathrm{~g} / \mathrm{L}$ & $15 \mathrm{mg} / \mathrm{L}$ \\
\hline
\end{tabular}

The attenuation lengths of the four samples listed in Table 1 are shown in Fig. 1. They were measured with a cuvette of $2 \mathrm{~mm}$ optical path. The attenuation lengths of the sample B and sample D at $\lambda<$ $345 \mathrm{~nm}$ were not obtained because the transmission was close to 0. As shown in Fig. 1, the attenuation of the LAB is negligible at $\lambda>340 \mathrm{~nm}$. Moreover, the attenuation length curve of sample $\mathrm{C}$ overlaps with that of sample $\mathrm{D}$ at $\lambda>360 \mathrm{~nm}$ and crosses with the curve of sample B at $349 \mathrm{~nm}$. This denotes that bisMSB has a dominant contribution to the attenuation at $\lambda>349 \mathrm{~nm}$. At $\lambda<349 \mathrm{~nm}$, the attenuations by the LAB and PPO become prevalent.

Figure 2 shows the attenuation length of the ternary liquid scintillator sample D $(345 \mathrm{~nm}<\lambda<$ $417 \mathrm{~nm})$. Four cuvettes $(2 \mathrm{~mm}, 4 \mathrm{~mm}, 1 \mathrm{~cm}$ and $10 \mathrm{~cm}$ optical path) were used. The cuvettes of different optical paths provided a cross check with each other. The attenuation curves measured by the cu- vettes of different optical paths largely overlap. The attenuation lengths at longer wavelengths cannot be obtained because the transmissions even for the $10 \mathrm{~cm}$ optical path are close to $100 \%$, but this measurement can be performed by a long tube, which has been described in Ref. [4].

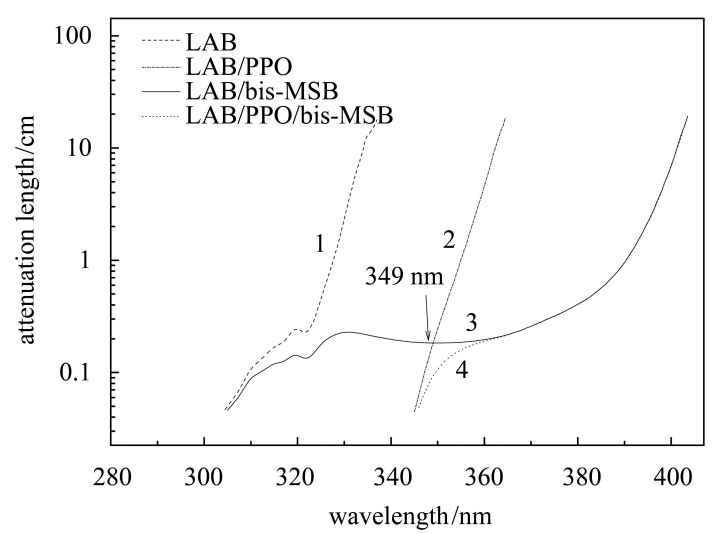

Fig. 1. The attenuation lengths of the pure LAB (1), sample B (2), sample C (3) and sample D (4).

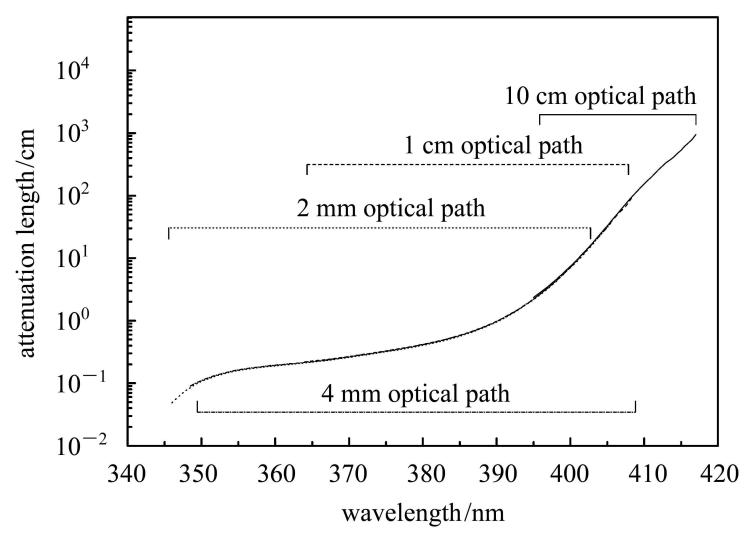

Fig. 2. The attenuation length of the ternary liquid scintillator sample D. The transmission measurements were performed with the $\mathrm{cu}^{-}$ vettes of $2 \mathrm{~mm}, 4 \mathrm{~mm}, 1 \mathrm{~cm}$ and $10 \mathrm{~cm}$ optical path.

Photons could be re-emitted after the absorption. The emission spectrum can be measured with a fluorescence spectrometer. In order to measure the intrinsic emission spectra of the LAB, the PPO and the bisMSB, we diluted them with cyclohexane, which can be regarded as transparent in our wavelength region of interest. Meanwhile, we prepared a $3 \mathrm{~g} / \mathrm{L} \mathrm{PPO}$ in cyclohexane to study the emission spectra at high concentration. These samples were placed in $2 \mathrm{~mm}$ cuvettes for the emission spectra measurements. To study the energy transfer between the PPO and the bis-MSB, a small amount of the ternary liquid scintillator sample D was painted on a glass surface and 
its emission spectrum was measured. The thickness of this sample is so small that the radiative energy transfer process (emission after absorption process) can be ignored and only the non-radiative process can occur in this thickness.

The LAB sample and the bis-MSB sample were excited by ultraviolet light at $280 \mathrm{~nm}$ and $360 \mathrm{~nm}$, respectively. The dilute PPO sample, the $3 \mathrm{~g} / \mathrm{L} \mathrm{PPO}$ sample and the ternary liquid scintillator sample were excited at $310 \mathrm{~nm}$. Most of the excitation energy was absorbed by the PPO in the ternary sample when they were excited at $310 \mathrm{~nm}$.

Figure 3 shows their emission spectra measured by a Fluorolog-3 fluorescence spectrometer. The emission spectra were measured in a front-face geometry to minimize the self-absorption of the fluorescence. We see from Fig. 3 that the emission spectra of the $3 \mathrm{~g} / \mathrm{L} \mathrm{PPO}$ and the dilute PPO show no significant difference in shape and peaks. This indicates that the formation of the transient dimers (see Ref. [11]) is not obvious, which changes the shape and peaks of the emission spectrum and lowers the fluorescence yield. Hence, the concentration quenching of the PPO is not important at the concentration of $3 \mathrm{~g} / \mathrm{L}$. It is also observed that the fluorescence of the bis-MSB was not present in the emission spectrum of the thin ternary liquid scintillator sample D. This shows that the nonradiative energy transfer process between the $\mathrm{PPO}$ and the bis-MSB is negligible. In the ternary liquid scintillator, the possible energy transfer process between the PPO and the bis-MSB is the absorption and re-emission process. In the next section, we will report the measurement of the re-emission probability of the PPO and the bis-MSB.

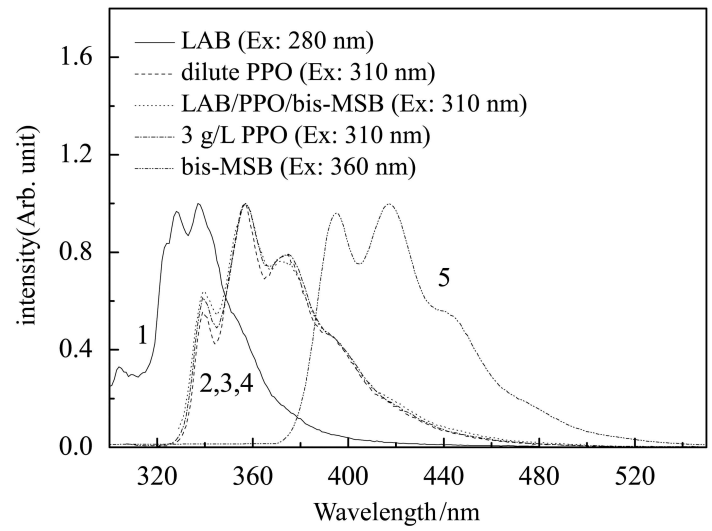

Fig. 3. The emission spectra of the dilute $\mathrm{LAB}$ (1), the dilute PPO (2), the $3 \mathrm{~g} / \mathrm{L}$ PPO (3), the ternary liquid scintillator sample D painted on the glass surface(4) and the dilute bis-MSB (5). The spectra were collected at a front-face geometry and all spectra shown in the figure are in arbitrary units.

\section{Fluorescence quantum yield mea- surement}

\subsection{Measure fluorescence quantum yield with relative method}

The fluorescence quantum yield is defined as the re-emission probability after an absorption. One of the practical methods to measure the fluorescence quantum yield is the relative method, which employs the fluorescence spectrometer to measure the fluorescence intensities of the unknown fluorescence quantum yield sample and the known fluorescence quantum yield sample (the reference sample). The expression for the unknown fluorescence quantum yield is $[12]$

$$
\Phi_{x}=\Phi_{r} \frac{B_{r}}{B_{x}} \frac{\int s_{x}(\lambda) \mathrm{d} \lambda}{\int s_{r}(\lambda) \mathrm{d} \lambda} \frac{n_{x}^{2}}{n_{r}^{2}},
$$

where the subscripts $r$ and $x$ refer to the reference sample and the unknown fluorescence quantum yield sample, $\Phi$ is the quantum yield, $s(\lambda)$ is the emission spectrum, the item $\left(n_{x} / n_{r}\right)^{2}$ is the correction of the refractive index effect and $B$ is the fraction of the excitation light absorbed by the sample, which is given by

$$
B=1-T
$$

where $T$ is the transmission of the sample. The transmission can be measured by an UV-Vis spectrophotometer.

\subsection{Samples}

The reliability of the fluorescence standard is quite important for the relative method. Quinine sulfate in $0.1 \mathrm{~mol} / \mathrm{L}$ sulfuric acid, whose fluorescence quantum yield is 0.55 [13], is an ideal fluorescence standard. It has been widely used as the fluorescence quantum yield standard. Its fluorescence quantum yield can be regarded to be independent of the excitation wavelength over 200-390 nm [12]. In order to have a cross check of our method and instrument, fluorescence quantum yield standard harmane in $0.1 \mathrm{~mol} / \mathrm{L}$ sulfuric acid was prepared as well. The fluorescence quantum yield of harmane is 0.83 [14].

As described in Section 2, the concentration quenching effect is not obvious in the $3 \mathrm{~g} / \mathrm{L} \mathrm{PPO}$ sample. The fluorescence quantum yield of the PPO when the PPO concentration is $3 \mathrm{~g} / \mathrm{L}$ should be equal to that in the lower concentration. It is reported that 
the fluorescence collection efficiency will change because of the drift in scintillation gravity when the absorption of the sample is high in the fluorescence intensity measurement (see Ref. [12]). The change in fluorescence collection efficiency will change the fluorescence intensity and bring great errors to the fluorescence quantum yields. We carefully calibrated this effect with the standard samples of different absorptions which were excited at the same wavelength, and we observed that the fluorescence intensities were proportional to the absorptions when they were lower than 0.5. We used the diluted samples with absorptions lower than 0.5 for both the samples and the references, thus the effect of the drift of scintillation gravity can be ignored. We diluted the PPO in cyclohexane to measure the fluorescence quantum yield of the PPO (the PPO concentration was 125 $\mathrm{mg} / \mathrm{L}$ ). Moreover, we prepared several LAB/bis-MSB samples (the bis-MSB concentrations were $1 \mathrm{mg} / \mathrm{L}$, $5 \mathrm{mg} / \mathrm{L}, 7.5 \mathrm{mg} / \mathrm{L}$ and $15 \mathrm{mg} / \mathrm{L}$, respectively) to measure the fluorescence quantum yield of the bis-MSB. As described in Section 1, the absorption of the LAB is negligible when the wavelength of excitation light is larger than $340 \mathrm{~nm}$ compared with the bis-MSB.

\subsection{Corrections and calibration}

In our measurements, the fluorescence spectra were corrected by the wavelength sensitivity curve of the photomultiplier and the variation of the intensity of the excitation light by the reference detector, which was mounted on the fluorescence spectrometer.

Figure 4 shows the integrated fluorescence intensities of unit absorption, i.e. $\int s_{r}(\lambda) \mathrm{d} \lambda / B_{r}$ in Eq. (2), as a function of the excitation wavelength of the two

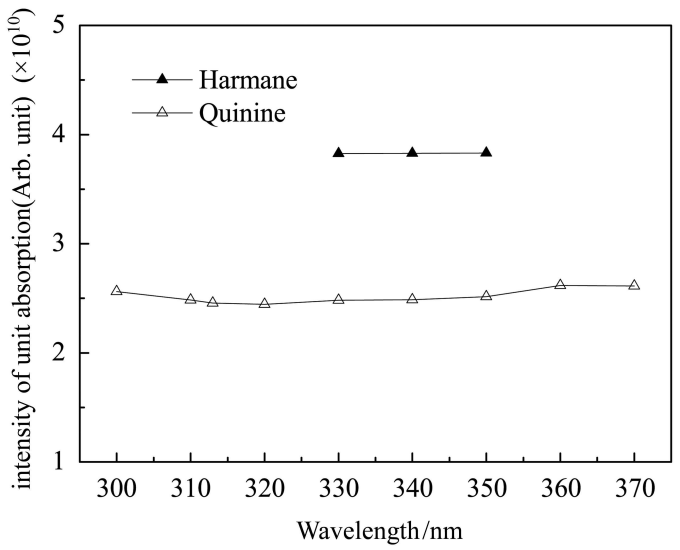

Fig. 4. The integration fluorescence intensities of unit absorption (i.e. $\int s(\lambda) \mathrm{d} \lambda / B$ ) in the Eq. (2) of the quinine sulfate sample and the harmine sample as a function of excitation wavelength. standard samples. These samples were placed in $2 \mathrm{~mm}$ cuvettes during the absorption and emission measurements and the fluorescence was collected in a frontface geometry during the fluorescence spectra measurements.

The intensity of unit absorption of the quinine sulfate, which is proportional to the fluorescence quantum yield, is flat within $2 \%$. The average intensities of unit absorption of the quinine sulfate sample and the harmane sample are $2.44 \times 10^{10}$ and $3.83 \times 10^{10}$, respectively. Since the fluorescence spectra of the two standard samples are similar and both of the samples are diluted, the refractive indices of the two standard samples can be regarded as equal. The ratio of the fluorescence quantum yields of the two standard samples can be obtained from Eq. (2). This value is 1.56. The ratio of the fluorescence quantum yields of the two standard samples which were reported in Refs. $[13,14]$ is $1.51(0.83 / 0.55)$.

\subsection{Results}

In the absorption and fluorescence intensity measurements, the PPO and the bis-MSB samples were placed in $2 \mathrm{~mm}$ cuvettes. The absorptions at different wavelengths were measured with the spectrophotometer. The fluorescence intensities were measured with the Fluorolog-3 spectrometer and the fluorescence was collected in the front-face geometry in order to minimize the self-absorption of the fluorescence. The excitation wavelength of the PPO sample varied from $300 \mathrm{~nm}$ to $340 \mathrm{~nm}$ and the bis-MSB from $360 \mathrm{~nm}$ to $380 \mathrm{~nm}$. Fig. 5 shows the fluorescence intensities at different absorptions and corresponding linear fits. Since the fluorescence quantum yields are

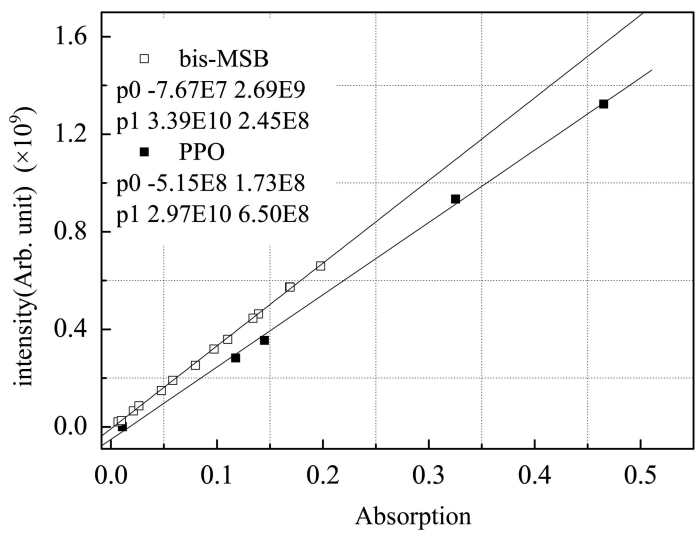

Fig. 5. The fluorescence intensities of the PPO and the bis-MSB samples at different absorptions. The excitation wavelength of the PPO sample was from 300 to $340 \mathrm{~nm}$ and bis-MSB from $360 \mathrm{~nm}$ to $380 \mathrm{~nm}$. The absorption is the $B$ described in Eq. (2). 
independent of excitation wavelength, the slopes of linear fits which denote the fluorescence intensities of unit absorption are proportional to their fluorescence quantum yields.

Table 2. The fluorescence quantum yield of the PPO and the bis-MSB.

\begin{tabular}{cc}
\hline material & fluorescence quantum yield \\
\hline PPO & 0.77 \\
bis-MSB & 0.96 \\
\hline
\end{tabular}

The average refractive indices of the samples were calculated by the expression $\int s(\lambda) n(\lambda) \mathrm{d} \lambda / s(\lambda) \mathrm{d} \lambda$, where $s(\lambda)$ and $n(\lambda)$ are the corresponding emission spectrum and the refractive index, respectively. The refractive index of the LAB was measured by Abbe prism and $\mathrm{V}$ prism methods. The average refractive index of the LAB for the bis-MSB fluorescence is 1.50 . The refractive index of the $0.1 \mathrm{~mol} / \mathrm{L}$ sulfate acid and the cyclohexane were obtained from Ref. [15] and Ref. [16]. Their average refractive indices are 1.34 and 1.44, respectively. The fluorescence quantum yields of the PPO and the bis-MSB can be obtained from Eq. (2) by comparing their fluorescence intensities of unit absorption with that of the quinine sulfate sample. Table 2 shows the results. Both of the fitting errors of the PPO and the bis-MSB fluorescence quantum yields are 0.03 .

\section{Conclusion}

The absorption and emission properties of a ternary liquid scintillator were studied. The absorption was dominant by LAB and PPO at wavelengths less than $349 \mathrm{~nm}$ and the absorption of bis-MSB become privilege at wavelength larger than $349 \mathrm{~nm}$. The fluorescence quantum yield of the PPO and the bisMSB were measured. This makes it possible to model the absorption and re-emission process in the studied ternary liquid scintillator.

\section{References}

1 Daya Bay Collaboration. arXiv:hep-ex/0701029v1

2 DING Y et al. Nucl. Instrum. Methods A, 2008, 584: 238243

3 Yeh M et al. Nucl. Instrum. Methods A, 2007, 578: 329339

4 LIU Jin-Chang et al. HEP \& NP, 2007, 30(1): 76-79 (in Chinese)

5 ZHONG W L et al. Nucl. Instrum. Methods A, 2008, 587: 300-303

6 XIAO H L, DENG J S. arXiv:nucl-ex/0904.1329v3
7 Birks J B. The Theory and Practice of Scintillation Counting Oxford, EnglandPergamon Press, 1964. 278-312

8 Cohen S G et al. Proc. Phys. Soc. B, 1956, 69: 593-605

9 D'Ambrosio C et al. Appl. Spectrosc., 1991, 45(3): 484-490

10 Martinho J M G, Maçanita A L, Berberan-Santos M N. J. Chem. Phys., 1989, 90(1): 53-59

11 Berlman I B. J. Chem. Phys., 1961, 34: 1083-1084

12 Demas J N et al. J. Chem. Phys., 1971, 75(8): 991-1023

13 Melhuish W H. J. Phys. Chem., 1961, 65: 229

14 Pardo A et al. Journal of Luminescence., 1992, 51: 269-274

15 Daimon M, Masumura A. Appl. Opt., 2007, 46: 3811-3820

16 Rheims J et al. Meas. Sci. Technol., 1997, 8: 601-605 